\title{
Investigación basada en el diseño en la formación inicial de docentes para una enseñanza integrada de la naturaleza de la ciencia y el pensamiento crítico
}

\author{
Cristina Cobo-Huesa (D), Ana M. Abril (iD , Marta R. Ariza (iD \\ Departamento de Didáctica de las Ciencias, Facultad de Humanidades y Ciencias de la Educación. \\ Universidad de Jaén. Campus Las Lagunillas, Jaén.España.cchuesa@ujaen.es, amabril@ujaen.es, \\ mromero@ujaen.es
}

[Recibido: 29 diciembre 2020. Revisado: 8 abril 2021. Aceptado: 13 mayo 2021]

\begin{abstract}
Resumen: Promover el conocimiento didáctico del contenido del profesorado en formación inicial para una enseñanza eficaz de la naturaleza de la ciencia y el pensamiento crítico es uno de los grandes retos a los que debe hacer frente la investigación educativa para mejorar la alfabetización científica de las futuras generaciones. Con el fin de ofrecer propuestas didácticas basadas en la investigación y evaluadas en el contexto real del aula, en este trabajo, se presenta la investigación enfocada al diseño de una propuesta de formación inicial de profesorado para una enseñanza integrada de estos dos constructos. El estudio ilustra el proceso de desarrollo y mejora basada en pruebas y aporta evidencias sobre su eficacia para obtener los resultados buscados, cuantificando el tamaño del efecto con un diseño pre-post test con grupo control.
\end{abstract}

Palabras clave: conocimiento didáctico del contenido, investigación basada en el diseño; naturaleza de la ciencia; pensamiento crítico; formación inicial de profesorado.

Design-based research in initial teacher training for an integrated teaching of nature of science and critical thinking

Abstract: Fostering preservice teachers' pedagogical content knowledge for effective teaching of the nature of
science and the promotion of critical thinking are both greater challenges for educational research, crucial for
improving future generations' scientific literacy. With the aim to provide research-based didactical proposals
evaluated in real classroom contexts, a design-based research approach was applied to develop an intervention for
initial teacher education, which addresses these aspects in an integrated way. The study shows the design
development and improvement based on evidence and its effectiveness to achieve the intended outcomes,
measuring the effect size through a pre-post research design with a control group.

Keywords: Pedagogical content knowledge; design-based research; nature of science; critical thinking; initial teacher training.

Para citar este artículo: Cobo-Huesa C., Abril A. M. y Ariza M. R. (2021) Investigación basada en el diseño en la formación inicial de docentes para una enseñanza integrada de la naturaleza de la ciencia y el pensamiento crítico. Revista Eureka sobre Enseñanza y Divulgación de las Ciencias 18(3), 3801. doi: 10.25267/Rev_Eureka_ensen_divulg_cienc.2021.v18.i3.3801

\section{Introducción}

Para la mejora de la alfabetización científica de las futuras generaciones, la Enseñanza de las Ciencias debe orientarse a mostrar la presencia e importancia de la ciencia y la tecnología en la vida cotidiana, y a la formación íntegra en conocimientos y habilidades para comprender y participar de manera fundamentada en asuntos socio-científicos (Forawi 2016, JiménezAleixandre y Puig 2012, Kötter y Hammann 2017).

En este contexto, la comprensión de la Naturaleza de la Ciencia $(\mathrm{NdC})$ y el desarrollo de las destrezas del Pensamiento Crítico (PC) se presentan como dos elementos clave (OCDE 2019, Turiman, Omar, Daud y Osman 2012, Vieira, Tenreiro-Vieira y Martins 2011, Yacoubian 2015). Sin embargo, la enseñanza eficaz de ambos constructos se ha visto mermada, en buena parte, por su compleja naturaleza. Este hecho reivindica la importancia de diseñar estrategias 
metodológicas en la formación inicial del profesorado, basadas en evidencias derivadas de la investigación en el contexto real del aula (Guisasola y Oliva 2020), que aporten información de utilidad en el ámbito del, aún limitado, Conocimiento Didáctico del Contenido (CdC) para la enseñanza de la $\mathrm{NdC}$ y el PC.

\section{Naturaleza de la Ciencia y Pensamiento Crítico en la Enseñanza de las Ciencias}

La $\mathrm{NdC}$ consiste en un meta-conocimiento sobre la ciencia, que contempla la fundamentación epistemológica del conocimiento y los procesos de la ciencia, así como los factores sociales que influyen en su construcción y validación (Acevedo-Díaz y García-Carmona 2015, Manassero-Mas y Vázquez-Alonso 2019). Por su parte, el PC se concibe como una habilidad cognitiva de orden superior encaminada a mejorar nuestro pensamiento a la hora de elaborar juicios y tomar decisiones sobre qué creer y hacer, para alcanzar nuestras metas con éxito (Ennis 1991, Halpern 2010, Zoller y Nahum 2012).

Las destrezas de PC, en estrecha relación con las del pensamiento científico (Vázquez-Alonso y Manassero-Mas 2018), fundamentan la construcción y validación del conocimiento científico, conocimiento clave en la comprensión de la NdC. Igualmente, la aplicación del PC en las estrategias de enseñanza sobre $\mathrm{NdC}$ (Yacoubian 2015) favorecen los procesos de reflexión tan reclamados en la literatura para que éstas sean efectivas (Abd-El-Khalick 2013).

Estos aspectos sugieren la pertinencia de trabajar PC y $\mathrm{NdC}$ de manera integrada en la Enseñanza de las Ciencias, evitando así mostrar la ciencia como un conjunto de productos finalizados, reflexionando sobre el origen del conocimiento y el grado en que este se justifica por evidencias o explicaciones teóricas (OCDE 2019). Este enfoque competencial invita al alumnado a participar en cuestiones controvertidas sobre ciencia y tecnología, que evidencian el complejo entramado de la empresa científica y contribuyen a su emancipación social (Jiménez-Aleixandre y Puig 2012).

\section{Obstáculos en la enseñanza de la Naturaleza de la Ciencia y el Pensamiento Crítico}

A pesar de la relevancia didáctica de integrar la $\mathrm{NdC}$ y el PC en la Enseñanza de las Ciencias, varios factores dificultan su aplicación eficaz en el aula.

Por un lado, existe una falta de consenso en torno a su conceptualización. Respecto a la NdC, durante la primera década de este siglo, la perspectiva que ha prevalecido en la bibliografía internacional es la llamada visión consenso, centrada en los aspectos epistemológicos. Para muchos autores, los aspectos sociológicos han sido atendidos de manera insuficiente, mermando la posibilidad de ofrecer una imagen más holística, real y funcional de la ciencia (Allchin 2011, Manassero-Mas y Vázquez-Alonso 2019). En cuanto al PC, destaca la gran variedad de marcos teóricos que lo definen, y que consideran distintas y variadas destrezas y actitudes o motivaciones (Ennis 1991, Forawi 2016, Halpern 2010, Valenzuela, Nieto y Muñoz 2014). Estas discrepancias han contribuido a que el profesorado los conciba como contenidos complejos (Acevedo-Díaz y García-Carmona 2015, Barak, Ben-Chaim y Zoller 2007), irrelevantes para la vida real, en el caso de la NdC (Kötter y Hammann 2017) o solo accesibles para estudiantes de alto desempeño académico, en el caso del PC (Zohar y Schwartzer 2005).

Por otro lado, existen obstáculos como la presencia implícita de estos dos constructos en el currículo (Borges-Fernandes, Pires y Delgado-Iglesias 2018, Vieira et al. 2011), la escasa formación sobre NdC (Cobo, Romero y Abril 2020, Demirdöğen, Hanuscin, UzuntiryakiKondakci y Köseoğlu 2016) y el limitado PC y conocimiento metacognitivo del profesorado (Qing, Jing y Yan 2010, Zohar y Schwartzer 2005). 


\section{Investigación basada en el diseño para orientar la formación inicial de profesorado}

Para introducir cambios de calidad en la educación científica, es imprescindible el desarrollo de propuestas de formación inicial de profesorado respaldadas en evidencias sólidas. Así, la investigación basada en el diseño o DBR (en inglés, Design Based Research) se presenta como una metodología adecuada para diseñar, desarrollar y evaluar propuestas en el contexto real educativo sobre aspectos de compleja naturaleza, como el CdC para la enseñanza de la $\mathrm{NdC}$ y PC (Guisasola y Oliva 2020, Plomp 2013, Romero-Ariza 2014).

Por tanto, siguiendo el marco de la DBR, en este trabajo se muestra el diseño de una propuesta de formación inicial de profesorado que tiene por objetivo mejorar su comprensión sobre la $\mathrm{NdC}$, el PC, y su CdC para la enseñanza integrada de ambos. Este diseño ha sido guiado bajo la siguiente pregunta de investigación: ¿Qué características ha de tener una propuesta de formación inicial de docentes que les prepare para una enseñanza integrada de la $\mathrm{NdC}$ y el PC?

\section{Metodología}

\section{Muestra y contexto del estudio}

La investigación sobre la eficacia de la propuesta se llevó a cabo con muestras de profesorado en formación inicial de tercer y cuarto curso del Grado de Educación Primaria de la Universidad de Jaén. El estudio incluyó un análisis pre-post test con grupo control. El grupo experimental contó con 101 estudiantes (58 mujeres) en el primer ciclo (ver Figura 1), y con 118 estudiantes (64 mujeres) en el segundo. La moda de edad era de 20 y 21 años, respectivamente. El grupo control estaba constituido por 30 estudiantes (18 mujeres) en el primer ciclo. En el segundo ciclo, este grupo lo constituían 72 estudiantes (39 mujeres) en el pre-test y 37 estudiantes (25 mujeres) en el post-test (moda de edad: 20 años en ambos grupos). El $76 \%$ de los estudiantes del grupo experimental y el $75 \%$ del grupo control no cursaron el Bachillerato de formación científica, previo a su ingreso a la universidad, y ninguno había recibido enseñanza explícita previa sobre NdC, PC o CdC.

La propuesta fue implementada en el contexto de las prácticas de las asignaturas sobre educación científica de la titulación Didáctica de las Ciencias de la Naturaleza I y Didáctica de las Ciencias de la Naturaleza II, de tercer y cuarto curso, respectivamente. El currículo de estas asignaturas contempla, entre otros, conocer tendencias actuales en la enseñanza de las Ciencias de la Naturaleza y el dominio de los elementos curriculares de Educación Primaria, entre los cuáles encontramos como contenidos, aspectos sobre la $\mathrm{NdC}$ y destrezas competenciales asociadas al PC (Borges-Fernandes et al. 2018). Las autoras implementaron la propuesta en los grupos experimentales. El grupo control recibió una formación tradicional.

\section{Fases de la investigación}

Partiendo del consenso generalizado sobre DBR (Romero-Ariza 2014), la investigación para el diseño se desarrolló en tres grandes fases: investigación preliminar (1), desarrollo y pilotaje (2) y evaluación final y principios de diseño (3) (Figura 1). 


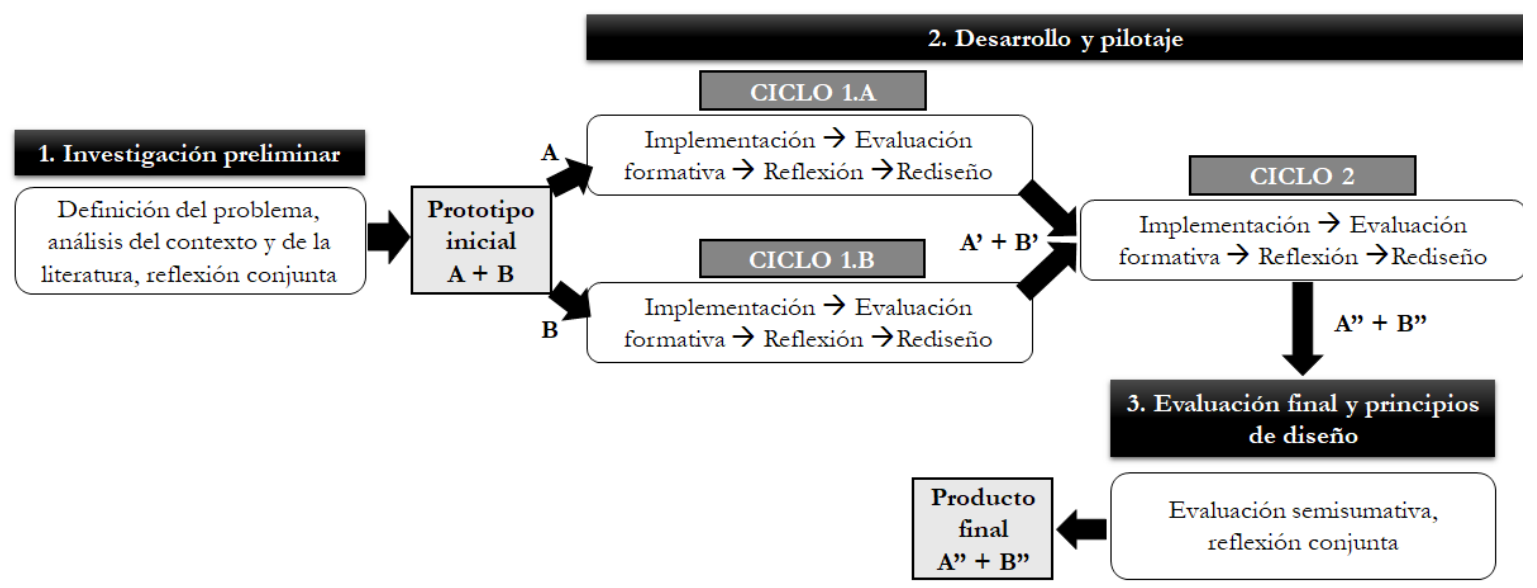

Figura 1. Fases de la DBR de la propuesta de formación inicial de profesorado

A continuación, se detalla la concreción de cada fase en el contexto del estudio.

\section{Fase 1-Investigación preliminar}

El equipo docente-investigador definió el problema, revisó la literatura al respecto y, considerando el contexto del estudio, consensuó los principios metodológicos que guiaron el diseño del prototipo de la propuesta (principios de diseño), y que serían validados y/o refinados en la fase de desarrollo y pilotaje (Romero-Ariza 2014). La identificación de principios de diseño, entendidos como las características de una secuencia de enseñanza y aprendizaje (SEA) que surgen de la investigación para informar las actividades de diseño (Plomp 2013), es esencial en la construcción de acciones de aula y se convierten en el punto de partida de nuevas propuestas.

Fase 2-Desarrollo y pilotaje

En esta fase se llevaron a cabo dos ciclos iterativos de implementación, evaluación, reflexión y rediseño del prototipo de la propuesta (Plomp 2013, Romero-Ariza 2014) distribuidos en el tiempo de manera lineal: ciclo 1 entre febrero de 2018 y enero de 2019 (1.A y 1.B antes y después de las vacaciones estivales respectivamente, en dos cuatrimestres diferentes); ciclo 2 entre febrero y mayo de 2019 (Figura 1).

Durante el ciclo 1, se desarrolló la propuesta en un mismo grupo de estudiantes, pero de manera interrumpida. Así, primero se implementó y evaluó la parte A de la propuesta (ciclo 1.A), enfocada a la mejora de la comprensión de la $\mathrm{NdC}$ y el desarrollo del PC y, después, la parte B (ciclo 1.B), orientada a trabajar el CdC de ambos. Esta división temporal se justifica, por un lado, porque, si bien una enseñanza sobre NdC y PC no es suficiente para mejorar su $\mathrm{CdC}$, el desarrollo de los primeros es indispensable para abordar este último (Demirdöğen et al. 2016, Kadir 2017). Por otro lado, abordar el CdC es una ardua tarea (Nilsson y Loughran 2012), lo que llevó al equipo a desarrollar y pilotar la parte B en un cuatrimestre posterior al abordaje de la parte A.

En el ciclo 2, se pilotó la propuesta completa en un solo cuatrimestre, con un nuevo grupo de estudiantes, incorporando las mejoras derivadas del ciclo anterior.

Tras cada ciclo, los cambios introducidos en la propuesta para su mejora se orientaron mediante una evaluación formativa, dirigida a valorar el proceso de enseñanza-aprendizaje, más allá de los resultados finales de aprendizaje (Guisasola y Oliva 2020). Para ello, se recurrió a los productos del alumnado, la observación y los registros anecdóticos. Estos permitieron recoger sus dificultades e inquietudes durante el trabajo autónomo, debates y puestas en común, haciéndoles partícipes del diseño (Romero-Ariza 2014). La evaluación sumativa de la 
eficacia de la propuesta se realizó a través de una evaluación pre-post test con grupo control de índole cuantitativo, combinado con el estudio cualitativo de casos en el grupo experimental.

Esta fase de desarrollo y pilotaje finalizó cuando se alcanzó un balance adecuado entre los objetivos previstos y los resultados alcanzados, de acuerdo a las percepciones recogidas en la evaluación formativa, y al análisis cuantitativo. En este último caso, la eficacia de la propuesta se evaluó a través del tamaño del efecto (TE), estadístico recomendado en diseños pre-post test con grupo control para valorar la magnitud del efecto de intervenciones didácticas teniendo en cuenta el progreso de los grupos experimental y control (Furtak, Seidel, Iverson y Briggs 2012, Morris 2008). Se consideró un TE relevante si su valor era igual o mayor a 0,30 (Cobo et aı. 2020).

\section{Fase 3-Evaluación finaly principios de diseño}

Finalmente, para valorar el impacto de la propuesta en el desarrollo profesional del profesorado en formación inicial, se llevó a cabo una evaluación, principalmente de carácter sumativo (McKenney 2001), pero que mantuvo los elementos de la evaluación formativa. Tras la reflexión derivada de dichos resultados, se definió el diseño final de la propuesta, se valoró la adecuación de los principios de diseño contemplados inicialmente y se extrajeron unos principios tentativos adicionales a modo de sugerencias de diseño para futuras propuestas educativas en la formación inicial de docentes.

\section{Resultados y discusión}

A continuación, se presentan los resultados que nos permiten dar respuesta a la pregunta de investigación acerca de qué características ha de poseer una SEA que nos permita preparar a docentes en formación inicial para una enseñanza integrada de la NdC y el PC.

\section{Fase 1-Investigación preliminar y diseño del prototipo}

En primer lugar, se identificó la necesidad de SEAs que trabajaran NdC y PC conjuntamente y su CdC. En base a ello, se revisaron trabajos basados en el análisis de las diferentes conceptualizaciones de $\mathrm{NdC}, \mathrm{PC}$ y $\mathrm{CdC}$, así como de intervenciones didácticas enfocadas a mejorar estos tres elementos en la formación inicial de profesorado. A continuación, se indican los principios de diseño extraídos de las recomendaciones de la literatura, y que guiaron la elaboración del prototipo de la propuesta:

- Adopción de una conceptualización holística de la NdC para atender y evaluar equitativamente las dimensiones epistemológica y sociológica de la ciencia (ManasseroMas y Vázquez-Alonso 2019).

- Adopción de la sintética taxonomía de PC propuesta por Halpern (2010), que, además, implica una evaluación contextualizada en situaciones cotidianas, lo que favorece su reconocimiento y aplicación fuera del contexto educativo (Halpern 2014).

- Adopción del enfoque motivacional para evaluar la dimensión emocional del PC, al ofrecer una mejor explicación de la variabilidad en su desempeño. La motivación depende de las expectativas de realizar una tarea satisfactoriamente y del valor asignado a pensar críticamente (Valenzuela et al. 2014).

- Adopción del marco teórico de CdC de Loughran, Bell y Mulhall (2012). Este integra la Representación del Contenido (ReCo) como instrumento para explicitar el conocimiento, creencias y razonamiento pedagógicos, de especial utilidad en formación inicial de profesorado (Hume y Berry 2011, Nilsson y Loughran 2012). 
- Formación previa en NdC (Demirdöğen et al. 2016) y PC (Kadir 2017) al abordaje del $\mathrm{CdC}$, pues este último requiere una comprensión rica de los contenidos a impartir (Loughran et al. 2012).

- Desarrollo de una instrucción explícita y reflexiva de la NdC (Abd-El-Khalick 2013), el PC (Marin y Halpern 2011) y el CdC (Loughran et al. 2012).

- Adopción de un enfoque socio-constructivista para favorecer la comprensión de rasgos de la NdC (Ozgelen, Yilmaz-Tuzun y Hanuscin 2011), el desarrollo del PC (Abrami et al. 2015) y el CdC (Hume y Berry 2011).

- Desarrollo de estrategias de enseñanza sobre NdC y PC en contextos científicos para ofrecer una visión coherente y práctica de ambos (Bailin 2002, Clough 2018). Esta contextualización cobra especial sentido cuando se sitúa en escenarios de aprendizaje auténticos, de la vida cotidiana (Abrami et al. 2015, Allchin 2011, Barak et al. 2007, Clough 2018, Valenzuela et al. 2014).

- Empleo de metodologías basadas en la indagación y en la historia de la ciencia para trabajar la NdC y el PC, pues implican aspectos relacionados con la generación y validación del conocimiento científico en su auténtico contexto socio-histórico (AbdEl-Khalick 2013, Allchin, Andersen y Kielsen 2014, Bailin 2002, McComas 2011).

Por consiguiente, la propuesta formativa se orientó a la construcción social del conocimiento en pequeños grupos cooperativos y a la contextualización del aprendizaje en escenarios de indagación basados en la historia de la ciencia y en aspectos de la vida cotidiana, como la aparición de moho en la fruta y la hidratación de legumbres. Siguiendo este enfoque socioconstructivista, el papel docente fue el de facilitar los contextos y medios necesarios para posibilitar el aprendizaje, así como el de formular cuestiones para la reflexión explícita sobre los rasgos de la $\mathrm{NdC}$ y las destrezas de PC.

El prototipo de la propuesta, se estructuró en dos grandes partes:

A. Comprensión de la NdC y desarrollo del PC: con la implementación de dos SEAs, la primera de ellas contextualizada en la historia de la ciencia sobre la teoría de la generación espontánea (presentada en Cobo-Huesa, Abril y Ariza 2019), y la segunda, en una indagación en el laboratorio orientada a minimizar los costes y el tiempo necesarios para hidratar legumbres. Cada SEA constaba de tres sesiones de dos horas. Ambas contemplaban espacios para la reflexión explícita y discusión en pequeño y gran grupo sobre rasgos de la $\mathrm{NdC}$, por ejemplo: «¿Tus compañeros de grupo y tú explicasteis inicialmente la aparición de moho en la naranja de la misma manera? ¿Por qué?» (carga teórica de las observaciones), «¿Qué característica destacaríais de Pasteur sobre Spallanzani y Needham? ¿Basta con ser objetivo para hacer mejor ciencia?» (características de los/as científicos/as). Del mismo modo, en relación a las destrezas de PC, se abordaron preguntas como las siguientes: «¿Qué cambio(s) propondríais en el diseño experimental de Van Helmont para comprobar si la ropa sucia era la causa de la aparición de los ratones?» (diferencia entre correlación y causa-efecto), «¿Cómo podemos reducir los errores durante nuestro experimento?» (réplicas y control experimental).

B. Enseñanza integrada de la NdC y el PC en las Ciencias de la Naturaleza: dirigida a trabajar el CdC para la enseñanza de la $\mathrm{NdC}$ y el PC en un contexto científico, y realizada en dos tareas de dos horas cada una. La primera tarea, estuvo enfocada a identificar, en pequeños grupos, los contenidos trabajados en un repositorio de SEAs elaboradas por el equipo docente, a modo de propuestas didácticas que muestran la enseñanza conjunta de contenidos disciplinares, rasgos de la $\mathrm{NdC}$ y destrezas de PC en diferentes contextos, así como a reflexionar sobre el CdC de estos a través de cuestiones de la ReCo (Loughran et al. 2012). A modo de ejemplo, una de dichas SEAs mostraba el empleo de la literatura infantil (cuento del 
patito feo) para trabajar la biodiversidad (contenido disciplinar), el papel de los esquemas de clasificación para comprender la naturaleza (rasgo de la $\mathrm{NdC}$ ) y la argumentación para defender clasificaciones (destreza de PC). La segunda tarea consistía en la cumplimentación individual de una ReCo, que explicitara el $\mathrm{CdC}$ del alumnado sobre un contenido disciplinar del currículo, un rasgo de la $\mathrm{NdC}$ y una destreza de $\mathrm{PC}$, estos dos últimos a elegir de un listado elaborado en base a las taxonomías de NdC y PC de Manassero-Mas y Vázquez-Alonso (2019) y Halpern (2010), respetivamente.

Para evaluar la eficacia de la propuesta, además de los instrumentos cualitativos (notas, observaciones y producciones escritas), se concretaron los cuestionarios cuantitativos a administrar como parte de la evaluación pre-post test con grupo control: diez ítems (Cobo et al. 2020) del Cuestionario de Opiniones sobre Ciencia, Tecnología y Sociedad (VázquezAlonso, García-Carmona, Manassero-Mas y Bennásar-Roig 2013), cinco situaciones sobre comprobación de hipótesis del test de Halpern (2010) y la escala motivacional de PC (Valenzuela, Nieto y Saiz 2011).

\section{Fase 2-Desarrollo y pilotaje}

A lo largo de esta segunda fase, se llevó a cabo la implementación, evaluación formativa y rediseño del prototipo de la propuesta. A continuación, se describen los aspectos más relevantes que dirigieron su refinamiento en cada ciclo.

\section{Primer ciclo}

En la primera parte del primer ciclo de refinamiento, dirigido a desarrollar y evaluar la parte A de la propuesta (Ciclo 1.A en la Figura 1), destacó positivamente el interés e implicación del alumnado. Esto se debió a la contextualización de las SEAs en aspectos cotidianos, sobre los que el alumnado reconoció «nunca haberse parado a pensar» (origen de moho en la fruta y optimización de la hidratación de legumbres). Sin embargo, encontró dificultades durante la indagación, requiriendo de una mayor interacción con las docentes, fundamentalmente, en el diseño experimental.

Por otro lado, del análisis de los productos de la SEA sobre la generación espontánea, se evidenciaron limitaciones para dar una respuesta fundamentada a preguntas relacionadas con la carga teórica de las observaciones y otros aspectos de la dimensión social de la $\mathrm{NdC}$ como la influencia de la sociedad en la ciencia o las características y motivaciones de los científicos (Cobo-Huesa et al. 2019).

En consecuencia, tras el ciclo 1.A, el equipo decidió incorporar una serie de modificaciones a la parte A de la propuesta:

- Incluyendo un breve contexto socio-histórico, previo a la presentación de los experimentos realizados en el seno de la controversia sobre el origen de los seres vivos, para favorecer la reflexión sobre la idoneidad de estos y la persistencia de la disputa.

- Incluyendo preguntas de metacognición previas al diseño experimental: «¿Qué vamos a analizar? ¿De qué materiales disponemos? ¿Qué estrategia vamos a seguir para comprobar nuestra hipótesis? ¿Cuántas placas vamos a utilizar y con qué fin? ¿Cómo podemos reducir errores? ¿Cómo podemos asegurar que los resultados obtenidos se deben a un aumento en la temperatura de cocción de las legumbres y no a otros factores? ¿Cómo vamos a analizar nuestros resultados?»

La segunda parte del primer ciclo de refinamiento, enfocado a evaluar la parte B de la propuesta (Ciclo 1.B en la Figura 1), evidenció las dificultades de los estudiantes para realizar la tarea grupal e individual sobre el CdC. Muchos de ellos manifestaron que, debido a su 
«limitada experiencia» docente, no podían dar respuesta a algunas preguntas, y consideraron la ReCo como un instrumento «abstracto y complejo».

Por tanto, tras el ciclo 1.B, se modificó la parte B del prototipo inicial de la propuesta:

- Incluyendo una sesión inicial de reflexión sobre la NdC y el PC, y su importancia curricular en la enseñanza de las Ciencias de la Naturaleza. Además, con el fin de relacionar las dos partes de la propuesta, esta sesión también contempló un breve repaso de las actividades de la parte A que trabajaban la NdC y el PC y, así hacer explícitos de nuevo los contenidos trabajados y el modo en que se integraron en las SEAs.

- Reduciendo el número de SEAs a analizar dentro del repositorio.

- Adoptando la ReCo propuesta por Nilsson y Loughran (2012) que integra la autoevaluación de la confianza en contestar a las cuestiones planteadas en una escala del 1-10, así como de su significatividad para planificar la enseñanza, con el fin de obtener información sobre los niveles de autoeficacia y creencias docentes del alumnado.

- Incluyendo el diseño de una propuesta didáctica por parte de los docentes en formación, como ayuda para guiar su planificación de aula plasmada en la ReCo.

- Administrando como pre-post test el cuestionario de creencias de eficacia en la enseñanza de las ciencias de Enochs y Riggs (1990) a los participantes, para evaluar la evolución de sus expectativas y percepciones de autoeficacia docente para promover resultados de aprendizaje positivos.

- Introduciendo la destreza de análisis de argumentos del test de Halpern, en la evaluación pre-post test.

Esta última modificación estuvo motivada, principalmente, por la observación del potencial de las SEAs de la parte A de la propuesta para el desarrollo de debates y, por tanto, para la elaboración y el análisis de argumentos en base a la calidad de las evidencias que los sustentan (Jiménez-Aleixandre y Puig 2012) y a la retroalimentación descrita entre las habilidades argumentativas y el desarrollo de visiones adecuadas sobre la NdC (McDonald y McRobbie 2012). En base a lo anterior, se realizaron unas últimas adaptaciones en las actividades de la propuesta, con el fin de trabajar más en profundidad el análisis de argumentos. Estos cambios ilustran cómo el análisis formativo tras la implementación guía la modificación del diseño para acercarnos a la consecución de los objetivos planteados.

\section{Segundo ciclo}

En el segundo ciclo de refinamiento, se implementó la propuesta completa de manera ininterrumpida, obteniendo resultados más positivos y satisfactorios que en las diferentes partes del ciclo anterior.

En la parte A de la propuesta, las preguntas metacognitivas añadidas permitieron al alumnado realizar una planificación experimental más ordenada. Por otro lado, la introducción histórica acerca del régimen político y corrientes de pensamiento reinantes (creacionismo, evolucionismo), les ayudó a analizar los diseños experimentales y razonamientos de los científicos de la época y, en consecuencia, comprender mejor la influencia de las ideas imperantes sobre las observaciones científicas, tal y como se reflejó en los productos generados y conversaciones entabladas.

La continua reflexión explícita sobre la $\mathrm{NdC}$ y el PC contribuyó a su asimilación significativa a lo largo de la propuesta. La reflexión explícita sobre los rasgos de la $\mathrm{NdC}$ y las destrezas 
implicadas en la comprobación de hipótesis y el análisis de argumentos trabajados en la indagación sobre el origen del moho en la fruta, mejoró el análisis crítico posterior de la historia de la ciencia sobre la generación espontánea, pues el alumnado pudo recurrir a su propia experiencia previa. Igualmente, en la siguiente SEA sobre la hidratación de legumbres, el alumnado, en su gran mayoría, contempló un control experimental adecuado y prestó mayor atención al control de las variables de estudio, lo que les permitió establecer conclusiones consistentes.

Respecto a la parte B, la reflexión inicial introducida en el segundo ciclo, permitió a los participantes reconocer la contribución del conocimiento sobre la $\mathrm{NdC}$ y el PC al desarrollo competencial de los estudiantes de Educación Primaria y, por tanto, la importancia de trabajar estos elementos en las tareas posteriores. Igualmente, la inclusión en la última tarea individual del diseño de su propia propuesta didáctica, asociada a la ReCo a cumplimentar, supuso los cimientos para una reflexión más focalizada sobre su $\mathrm{CdC}$, al disponer de una referencia didáctica a la que remitirse. Como ejemplo de ello, en la Tabla 1 del Anexo se presentan las reflexiones de una estudiante a las preguntas de la ReCo sobre la intención, importancia didáctica y método de evaluación de un rasgo de la $\mathrm{NdC}$ y una destreza del PC, en el contexto de su propuesta para la enseñanza del sistema óseo. En ellas, destaca la intención de promover la reflexión explícita sobre el trabajo científico $(\mathrm{NdC})$ y los criterios seguidos en la clasificación de los huesos, fomentando la metacognición (PC). Así, se contempla la evaluación del esqueleto (contenido científico), junto con el razonamiento y justificaciones subyacentes a su elaboración, destacando la labor de la comunidad científica en el conocimiento que disponemos sobre nuestro organismo.

Sin embargo, no todo el profesorado en formación inicial integró explícitamente la $\mathrm{NdC}$ y/o el PC en sus propuestas, a pesar de que dicho aspecto fue resaltado en las instrucciones de la tarea. Una parte destacada no supo discernir entre la dimensión epistemológica de los procesos científicos y el propio proceso de hacer ciencia, uno de los principales obstáculos en la enseñanza de la NdC (Bell, Mulvey y Maeng 2012), tal y como, de manera representativa, se presenta en la Tabla 2 del Anexo.

Por último, también se identificó un limitado conocimiento disciplinar y pedagógico sobre los contenidos científicos de las Ciencias de la Naturaleza, que puede suponer un obstáculo para una planificación eficaz de la NdC y el PC (Abd-El-Khalick 2013, Bailin 2002).

Tras este segundo pilotaje, destacamos la importancia de trabajar de manera reflexiva y explícita los contenidos sobre $\mathrm{NdC}$ y PC, a través del análisis de SEAs que aborden su enseñanza. Así mismo, resaltamos la diferencia entre el conocimiento sobre la $\mathrm{NdC}$ y el desarrollo de procesos científicos que implican la aplicación del PC, a través discusiones en gran grupo, tras la resolución de las tareas. No obstante, se decidió mantener la estructura general de la propuesta debido a la satisfactoria actuación del alumnado durante su implementación, a su adecuación al contexto y planificación curricular de la asignatura de la titulación, así como a los resultados obtenidos de la evaluación sumativa de los cuestionarios aplicados antes y después de los ciclos de pilotaje 1.A y 2 (no en el ciclo 1.B, al estar orientada a trabajar el $\mathrm{CdC}$ ). En la Figura 2 se muestra el TE de la propuesta de formación en cada ciclo para las distintas variables de estudio. 


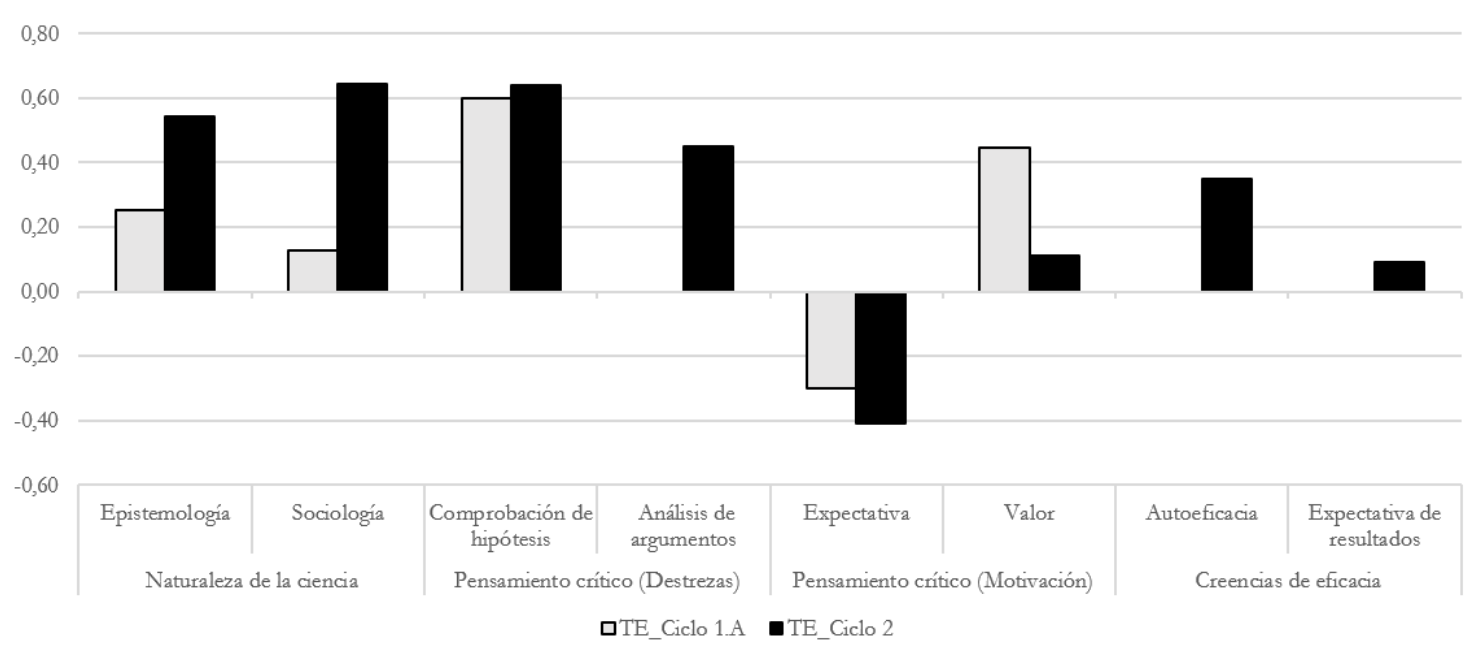

Figura 2. Tamaño del efecto de la propuesta formativa en el primer y segundo ciclo de desarrollo y pilotaje

De acuerdo a la Figura 2, tanto en el ciclo 1.A como en el ciclo 2 se obtuvieron TE positivos en prácticamente todas las variables, lo que avala la eficacia de la propuesta para promover una visión adecuada sobre la $\mathrm{NdC}$, el PC y las creencias de eficacia docentes.

En el ciclo 1.A, destacó la magnitud del efecto de la propuesta sobre la comprobación de hipótesis como destreza de PC (TE $\geq 0,30)$. Sin embargo, las expectativas de pensar críticamente de manera eficaz disminuyeron. Tal y como se ha explicado anteriormente, en este ciclo, no se evaluó el análisis de argumentos ni se administró el test de creencias de eficacia.

En el ciclo 2, se observó un TE relevante para las dos dimensiones de la $\mathrm{NdC}$, superior al del ciclo anterior, lo que apoya las percepciones de aula más satisfactorias detectadas en el segundo ciclo. Del mismo modo, para la dimensión de comprobación de hipótesis, el TE subió ligeramente, y además se detectó un TE relevante para la dimensión análisis de argumentos. En cambio, para el componente motivacional del PC se produjo una caída del TE para sus dos dimensiones, aunque este solo fue negativo en la referente a las expectativas, al igual que en el ciclo anterior. Este hecho sugiere el esfuerzo cognitivo que supone emplear el PC, pudiendo influir en la dimensión de expectativas cuando se trabaja explícitamente (Valenzuela et al. 2011). Por último, respecto a las creencias de autoeficacia, los resultados muestran un progreso en la autoeficacia y expectativas de resultados de los futuros docentes, con un TE relevante para la primera.

\section{Fase 3-Diseño final y principios de diseño}

En esta última fase, se llevó a cabo la evaluación semisumativa de la eficacia de la propuesta que, tras la reflexión conjunta del equipo docente-investigador, permitió la definición final de su diseño.

La Figura 3 representa el diseño final que responde a la pregunta de investigación planteada inicialmente. Este diseño es el resultado del pilotaje de un prototipo basado en la literatura especializada, que ha sido mejorado a través ciclos iterativos de implementación y evaluación formativa. La evaluación sumativa mediante un diseño pre-post test con grupo control, permite cuantificar los efectos de la SEA sobre el alumnado participante y aporta evidencias sobre la eficacia del diseño para conseguir los resultados perseguidos (Figura 2). 


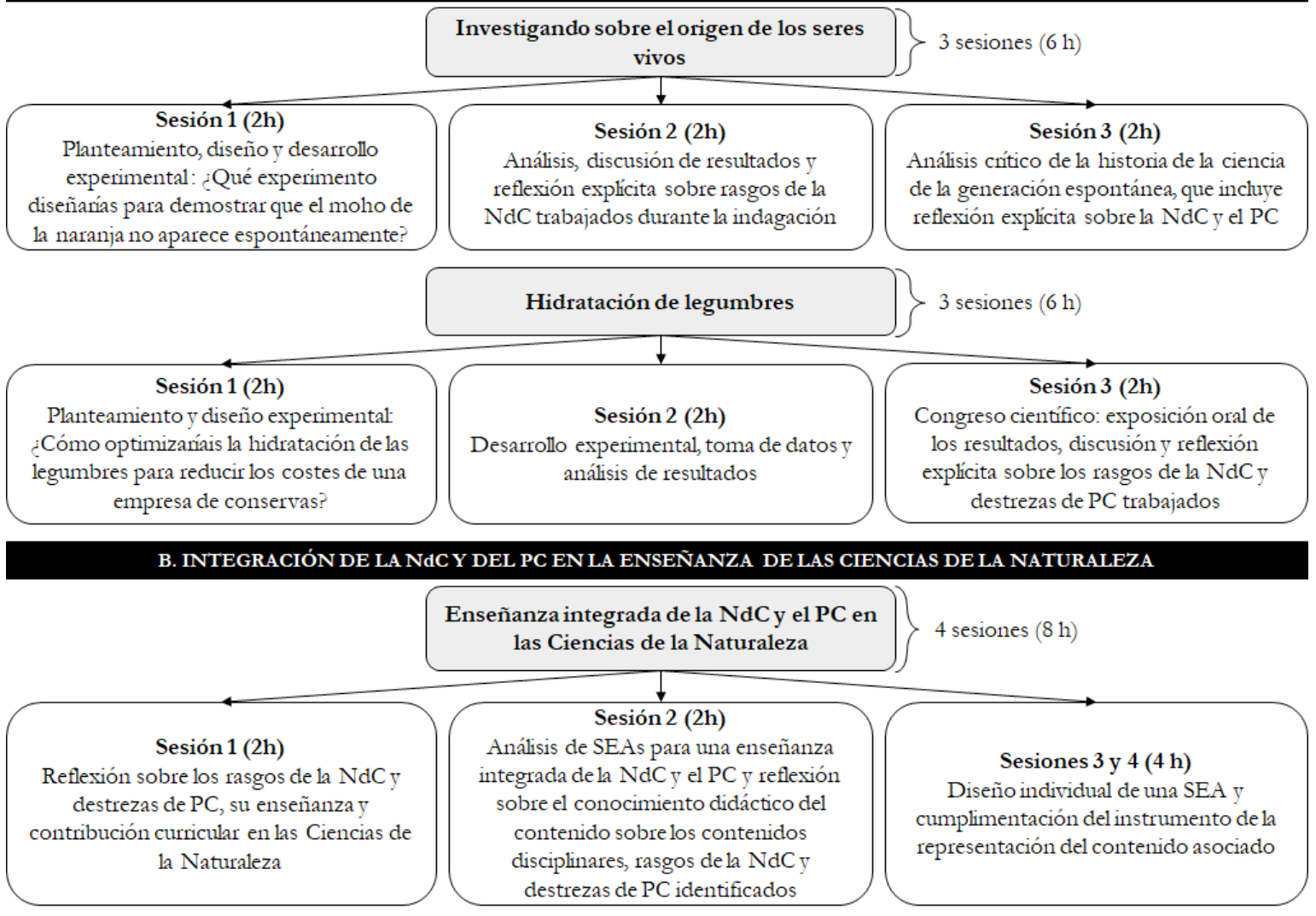

Figura 3. Propuesta final de formación inicial de profesorado para una enseñanza integrada de la NdC y el PC

Asimismo, siguiendo el fundamento de la DBR, los resultados obtenidos en el contexto del presente trabajo, sugieren la validación de los principios de diseño que guiaron el prototipo de la propuesta. Además, pudieron identificarse otros aspectos de gran interés por su contribución a la mejora de la propuesta y que se comentan a continuación.

En primer lugar, de la presente investigación emana la necesidad de una continua referencia a los contenidos sobre $\mathrm{NdC}$ y $\mathrm{PC}$, para favorecer su asimilación y reconocimiento en los diferentes contextos presentados. Este principio lo consideramos especialmente relevante en las primeras etapas de formación docente, donde predominan el desconocimiento e ideas erróneas sobre la NdC (Cobo et al. 2020, Demirdöğen et al. 2016) y la falta de conocimiento y aplicación del PC (Qing et al. 2010, Zohar y Schwartz 2005). De este modo, además, se favorece una construcción práctica real y funcional de estos constructos, al evidenciar su presencia en distintos ámbitos y procesos científicos.

En segundo lugar, la referencia a la contribución curricular de la $\mathrm{NdC}$ y del PC favoreció que el profesorado en formación inicial considerara su importancia en la enseñanza de las Ciencias de la Naturaleza. En este sentido, resultó fundamental destacar la aportación de la $\mathrm{NdC}$ al desarrollo de actitudes positivas hacia la ciencia y a la comprensión del origen y persistencia de diversas controversias socio-científicas (p.ej. influencia de la radiación móvil en la salud), así como del PC al desarrollo competencial del alumnado de Educación Primaria, su autonomía e independencia (Jiménez-Aleixandre y Puig 2012, Yacoubian 2015).

En tercer lugar, consideramos esencial contextualizar la construcción del CdC sobre $\mathrm{NdC}$ y PC del futuro profesorado a través del análisis de SEAs que muestren su enseñanza integrada en las Ciencias de la Naturaleza. De este modo, se pretende contribuir a la mejora de sus percepciones de autoeficacia, las cuales juegan un papel clave en definir estrategias, estimular 
su puesta en práctica (Park y Oliver 2008), y guiar su reflexión durante el diseño de sus propias SEAs y la cumplimentación de la ReCo.

En cuarto y último lugar, si bien autores como Hume y Berry (2011) proponen la construcción del CdC del profesorado en sus etapas más iniciales de formación colaborando con sus iguales, tras nuestro estudio, remarcamos también la importancia de que el alumnado se enfrente individualmente al diseño y planificación de sus propias propuestas didácticas. De este modo, el docente en formación podrá poner en juego su conocimiento y habilidades, enriquecidos con la cooperación previa, y su autorreflexión para identificar limitaciones y aspectos a mejorar. Este esfuerzo de autoevaluación personal quedó reflejado en las valoraciones de confianza parar satisfacer las cuestiones de la ReCo (Nilsson y Loughran 2012).

Estas características, fruto de la investigación desarrollada bajo el enfoque de la DBR, suponen principios de diseño tentativos que deben ser validados en futuros estudios.

\section{Conclusiones}

Este trabajo muestra cómo la aplicación sistemática de los principios de la DBR ha permitido afrontar con éxito el reto de diseñar una propuesta que responda a las carencias detectadas sobre el CdC para la enseñanza integrada de la $\mathrm{NdC}$ y el PC.

Siguiendo a McKenney (2001), el objetivo de la DBR debe ser contribuir al desarrollo profesional de los participantes, llevándose a cabo en colaboración «con» y no «para» ellos. En base a esto, las apreciaciones y dificultades manifestadas por el profesorado en formación inicial guiaron la mejora progresiva de la propuesta en los sucesivos ciclos de pilotaje. De modo que, la evaluación final permitió validar los principios de diseño que guiaron el prototipo de la propuesta y extraer unos principios de diseño tentativos, que sugieren su contribución al logro de los objetivos perseguidos, en el contexto de las necesidades reales de aula.

Así, por un lado, este trabajo viene a validar las recomendaciones pedagógicas de los expertos de la literatura: la combinación de aspectos sociológicos y epistemológicos para ofrecer una visión holística de la NdC (Allchin 2011, Manassero-Mas y Vázquez Alonso 2019), el enfoque explícito y reflexivo para una enseñanza eficaz de la NdC y el PC (Abd-El-Khalick 2013, Marin y Halpern 2011), la consideración de aspectos emocionales en la enseñanza para favorecer el compromiso en las tareas y apropiación de los contenidos (Allchin 2011, Valenzuela et al. 2014), la metacognición como herramienta para la mejora del PC (Abrami et al. 2015, Marin y Halpern 2011), la adopción de un enfoque socio-constructivista para favorecer la asimilación de los contenidos (Abrami et al. 2015, Hume y Berry 2011, Ozgelen et al. 2013), la combinación de enfoques metodológicos de probada eficacia (historia de la ciencia, controversias científicas, indagación y contextos significativos) (Acevedo-Díaz y García-Carmona 2015, Allchin et al. 2014, Bailin 2002) y el empleo de la ReCo para la construcción del CdC (Hume y Berry 2011, Nilsson y Loughran 2012).

Por otro lado, la evaluación de la propuesta a lo largo de los ciclos sugiere nuevas evidencias que se suman a las ofrecidas por la investigación especializada, y que se presentan como principios de diseño tentativos a ser validados en otros contextos de estudio. Estas nuevas evidencias subrayan la importancia de una enseñanza altamente reforzada, contextualizada y dinámica de la $\mathrm{NdC}$ y el PC, que potencie su carácter funcional y transversal, así como su contribución curricular. Del mismo modo, se reivindica una construcción contextualizada y social del $\mathrm{CdC}$, que también contemple espacios para su abordaje individual y la autorreflexión. 
El presente estudio también evidencia la necesidad de prestar más atención al componente motivacional del PC, dado su papel en la modulación del aprendizaje de las destrezas y su aplicación fuera del ámbito educativo (Halpern 2014, Valenzuela et al. 2011). En este sentido, la inclusión de espacios para que el alumnado haga balance de su aprendizaje y exprese sus dificultades, contribuiría a orientar de manera más eficaz los recursos necesarios para solventarlos y mejorar sus expectativas y desempeño cognitivos.

Para finalizar, es importante llamar la atención sobre el hecho de que, el análisis de los criterios de calidad asociados a la DBR revela la validez, utilidad y eficacia de este estudio (RomeroAriza 2014). Así pues, la implementación de la propuesta con diferentes cohortes de profesorado en formación inicial muestra que se trata de una propuesta factible de ser trasladada a contextos reales y a la actual práctica educativa. Igualmente, su eficacia para alcanzar los resultados educativos pretendidos, viene avalada por los datos cuantitativos que surgen de una metodología pre-post test con grupo control.

Por todo ello, esperamos que los resultados derivados de esta investigación basada en el diseño puedan arrojar luz para orientar futuras propuestas en la formación inicial de profesorado encaminadas a promover la enseñanza integrada de la $\mathrm{NdC}$ y el PC, aunando investigación y práctica educativa, en pos de la mejora de la calidad de la educación científica.

\section{Agradecimientos}

Proyecto de investigación "Educación de las Competencias Científica, Tecnológica y Pensamiento Crítico Mediante la Enseñanza de Temas de Naturaleza de Ciencia y Tecnología" (EDU2015-64642-R) (MINECO/FEDER), financiado por el Ministerio de Economía y Competitividad de España y el Fondo Europeo de Desarrollo Regional.

Ayuda predoctoral para la Formación de Personal Investigador, con cargo a la acción 4 del Plan de Apoyo a la Investigación de la Universidad de Jaén 2017-2019 (R5/04/2017).

\section{Referencias}

Abd-El-Khalick F. (2013) Teaching With and About Nature of Science, and Science Teacher Knowledge Domains. Science and Education 22, 2087-2107.

Abrami P.C., Bernard R.M., Borokhovski E., Waddington D.I., Wade C.A., Persson, T. (2015) Strategies for Teaching Students to Think Critically: A Meta-Analysis. Review of Educational Research 85(2), 275-314.

Acevedo-Díaz J.A., García-Carmona A. (2015) «Algo antiguo, algo nuevo, algo prestado». Tendencias sobre la naturaleza de la ciencia en la educación científica. Revista Eureka sobre Enseñanza y Divulgación de las Ciencias 13(1), 3-19. http://dx.doi.org/10.25267/Rev_Eureka_ensen_divulg_cienc.2016.v13.11.02

Allchin D. (2011) Evaluating knowledge of the nature of (whole) science. Science Education 95(3), 518-542.

Allchin D., Andersen H.M., Kielsen K. (2014) Complementary approaches to teaching nature of science: Integrating student inquiry, historical cases, and contemporary cases in classroom practice. Science Education 98(3), 461-486.

Bailin S. (2002) Critical Thinking and Science Education. Science and Education 11, 361-375.

Barak M., Ben-Chaim D., Zoller U. (2007) Purposely Teaching for the Promotion of Higherorder Thinking Skills: A Case of Critical Thinking. Research in Science Education 37, 353369. 
Bell R.L., Mulvey B.K., Maeng J.L. (2012) Beyond Understanding: Process Skills as a Context for Nature of Science Instruction. En M. Khine (Ed.), Advances in Nature of Science Research (pp. 225-246). Dordrecht: Springer.

Borges-Fernandes I.M., Pires D.M., Delgado-Iglesias J. (2018) ¿Qué mejoras se han alcanzado respecto a la Educación Científica desde el enfoque Ciencia-Tecnología-SociedadAmbiente en el nuevo Currículo Oficial de la LOMCE de $5^{\circ}$ y $6^{\circ}$ curso de Primaria en España? Revista Eureka sobre Enseñanza y Divulgación de las Ciencias 15(1), 1101. https://doi.org/10.25267/Rev_Eureka_ensen_divulg_cienc.2018.v15.11.1101

Clough M.P. (2018) Teaching and Learning About the Nature of Science. Science and Education 27, 1-5. https://doi.org/10.1007/s11191-018-9964-0

Cobo C., Romero M., Abril A. (2020) Indagación reflexiva e historia de la ciencia para construir una visión adecuada sobre la naturaleza de la ciencia en formación inicial de profesorado. Tecné, Episteme y Didaxis: TED 48, 13-31. https://doi.org/10.17227/ted.num48-10934

Cobo-Huesa, C., Abril, A.M. y Ariza, M.R. (2019) Propuesta didáctica en la formación de profesorado para trabajar naturaleza de la ciencia y pensamiento crítico. Ápice. Revista de Educación Cientifica 3(1), 15-28. https://doi.org/10.17979/arec.2019.3.1.4630

Demirdöğen B., Hanuscin D.L., Uzuntiryaki-Kondakci E., Köseoğlu F. (2016) Development and nature of preservice chemistry teachers' pedagogical content knowledge for nature of science. Research in Science Education 46, 575-612.

Ennis R. (1991). Critical thinking: a streamlined conception. Teaching Philosophy 14(1), 5-24.

Enochs L.G., Riggs I.M. (1990) Further development of an elementary science teaching efficacy belief instrument: A preservice elementary scale. School Science and Mathematics 90(8), 694-706.

Forawi S.A. (2016) Standard-based science education and critical thinking. Thinking skills and creativity 20, 52-62.

Furtak E.M., Seidel T., Iverson H., Briggs D.C. (2012) Experimental and Quasi-Experimental Studies of Inquiry-Based Science Teaching: A Meta-Analysis. Review of Educational Research 82(3), 300-329.

Guisasola J., Oliva J.M. (2020) Nueva sección especial de REurEDC sobre investigación basada en el diseño de secuencias de enseñanza. Revista Eureka sobre Enseñanza y $\begin{array}{llll}\text { Divulgación le Ciencias } & \text { 17(3), } & \end{array}$ https://doi.org/10.25267/Rev_Eureka_ensen_divulg_cienc.2020.v17.i3.3001

Halpern D.F. (2014) Thought and Knowledge: An Introduction to Critical Thinking ( $5^{\circ} \mathrm{Ed}$.). New York: Psychology Press.

Halpern D.F. (2010) The Halpern critical thinking assessment: Manual. Vienna: Schuhfried.

Hume A., Berry A. (2011) Constructing CoRes-a Strategy for Building PCK in Pre-service Science Teacher Education. Research in Science Education 41, 341-355.

Jiménez-Aleixandre M.P., Puig B. (2012) Argumentation, Evidence Evaluation and Critical Thinking. En B. Fraser, K. Tobin, C.J. McRobbie (Eds.), Second International Handbook of Science Education (pp. 1001-1015). Dordrecht: Springer.

Kadir M.A.A. (2017) What teacher knowledge matters in effectively developing critical thinkers in the 21st century curriculum? Thinking Skills and Creativity 23, 79-90. 
Kötter M., Hammann M. (2017) Controversy as a Blind Spot in Teaching Nature of Science Why the Range of Different Positions Concerning Nature of Science Should Be an Issue in the Science Classroom. Science and Education 26, 451-482.

Loughran J., Berry A., Mulhall P. (2012) Understanding and Developing Science Teacher's Pedagogical Content Knowledge. Rotterdam: Sense Publishers.

Marin L.M., Halpern D.F. (2011) Pedagogy for developing critical thinking in adolescents: Explicit instruction produces greatest gains. Thinking Skills and Creativity 6(1), 1-13.

Manassero-Mas M.A., Vázquez-Alonso A. (2019) Conceptualización y taxonomía para estructurar los conocimientos acerca de la ciencia. Conceptualización y taxonomía paraestructurar los conocimientos acerca de la ciencia. Revista Eureka sobre Enseñanza y $\begin{array}{llll}\text { Divulgación de las } 3104 . & \text { Ciencias }\end{array}$ https://doi.org/10.25267/Rev_Eureka_ensen_divulg_cienc.2019.v16.i3.3104

McComas W.F. (2011) The history of science and the future of science education: a typology of approaches to history of science in science instruction. En P.V. Kokkotas, K.S. Malamitsa, A.A. Rizaki (Eds.), Adapting Historical Knowledge Production to the Classroom (pp. 37-53). Rotterdam: Sense Publishers.

McDonald C.V., McRobbie C.J. (2012) Utilising argumentation to teach nature of science. En B.J. Fraser, K. Tobin, C.J. McRobbie (Eds.), Second International Handbook of Science Education (pp. 969-986). Dordrecht: Springer.

McKenney S. (2001) Computer-Based Support for Science Education Materials Developers in Africa: Exploring Potentials (Tesis doctoral inédita). University of Twente, Enschede, Países Bajos.

Morris S.B. (2008) Estimating effects sizes from pretest-posttest-control group designs. Organizational Research Methods 11(2), 364-386.

Nilsson P., Loughran J. (2012) Exploring the development of pre-service science elementary teachers' pedagogical content knowledge. Journal of Science Teacher Education 23, 699-721.

OCDE (2019) PISA 2018 Assessment and Analytical Framework. Paris: PISA OECD Publishing. https://doi.org/10.1787/b25efab8-en

Ozgelen S., Yilmaz-Tuzun O., Hanuscin D.L. (2013) Exploring the Development of Preservice Science Teachers' Views on the Nature of Science in Inquiry-Based Laboratory Instruction. Research in Science Education 43(3), 1551-1570.

Park S., Oliver J.S. (2008) Revisiting the Conceptualisation of Pedagogical Content Knowledge (PCK): PCK as a Conceptual Tool to Understand Teachers as Professionals. Research in Science Education 38, 261-284.

Plomp T. (2013) Educational Design Research: An Introduction. En T. Plomp, N. Nieveen (Eds.), Educational Design Research (pp. 9-35). Enschede: SLO.

Qing Z., Jing G., Yan W. (2010) Promoting preservice teachers' critical thinking skills by inquiry-based chemical experiment. Procedia-Social and Behavioral Sciences 2, 4597-4603. https://doi.org/10.1016/j.sbspro.2010.03.737

Romero-Ariza M. (2014). Uniendo investigación, política y práctica educativas: DBR, desafíos y oportunidades. magis, Revista Internacional de Investigación en Educación 7(14), 159-176. https://www.redalyc.org/pdf/2810/281032883012.pdf 
Turiman P., Omar J., Daud A.M., Osman K. (2012) Fostering the 21st Century Skills through Scientific Literacy and Science Process Skills. Procedia-Social and Behavioral Sciences 59, 110-116. https://doi.org/10.1016/j.sbspro.2012.09.253

Valenzuela J., Nieto A.M., Muñoz C. (2014) Motivación y disposiciones: enfoques alternativos para explicar el desempeño de habilidades de pensamiento crítico. Revista Electrónica de Investigación Educativa 16(3), 16-32. http://redie.uabc.mx/vol16no3/contenidovalnieto.html

Valenzuela J., Nieto A.M., y Saiz C. (2011) Critical thinking motivational scale (CTMS): una aportación para el estudio de la relación entre el pensamiento crítico y la motivación. Electronic Journal of Research in Educational Psychology 9(2), 823-848. http://dx.doi.org/10.25115/ejrep.v9i24.1475

Vázquez-Alonso A., García-Carmona A., Manassero-Mas M.A., Bennàsar-Roig, A. (2013) Science Teachers' Thinking About the Nature of Science: A New Methodological Approach to Its Assessment. Research in Science Education 43, 781-808.

Vázquez-Alonso A., Manassero-Mas M.A. (2018) Más allá de la comprensión científica: educación científica para desarrollar el pensamiento. Revista Electrónica de Enseñanza de las Ciencias 17(2), 309-336. http://reec.uvigo.es/volumenes/volumen17/REEC_17_2_02_ex1065.pdf

Vieira R.M., Tenreiro-Vieira C., Martins I.P. (2011) Critical thinking: Conceptual clarification and its importance in science education. Science Education International 22(1), 43-54. https://files.eric.ed.gov/fulltext/EJ941655.pdf

Yacoubian H.A. (2015) A framework for guiding future citizens to think critically about nature of science and socioscientific issues. Canadian Journal of Science, Mathematics and Technology Education 15(3), 248-260.

Zohar A., Schwartzer N. (2005) Assessing teachers' pedagogical knowledge in the context of teaching higher-order thinking. International Journal of Science Education 27(13), 15951620.

Zoller U., Nahum T.L. (2012) From teaching to KNOW to learning to THINK in science education. En B.J. Fraser, K.G. Tobin, C.J. McRobbie (Eds.), Second International Handbook of Science Education (pp. 209-229). Dordrecht: Springer. 
Anexo I. Ejemplos representativos de las Representaciones del Contenido (ReCos) elaboradas por el profesorado en formación inicial de Educación Primaria

Tabla 1. Reflexiones de una estudiante sobre la intención e importancia didáctica y métodos de evaluación de un rasgo de la $\mathrm{NdC}$ y una destreza del $\mathrm{PC}$ en el $3^{\circ}$ ciclo de Educación Primaria.

\begin{tabular}{|c|c|c|}
\hline & Rasgo de la NdC & Destreza del PC \\
\hline & $\begin{array}{l}\text { Para mejorar la comprensión de la naturaleza, } \\
\text { los/as cientificos/as elaboran esquemas de } \\
\text { clasificación. }\end{array}$ & $\begin{array}{l}\text { Identificar patrones comunes y establecer criterios } \\
\text { de clasificación. }\end{array}$ \\
\hline & Respuesta & Respuesta \\
\hline $\begin{array}{l}\text { ¿Qué pretendes } \\
\text { que aprendan los } \\
\text { estudiantes sobre } \\
\text { estos contenidos? }\end{array}$ & $\begin{array}{l}\text { «Como docente, quiero que el alumnado } \\
\text { aprenda la manera de trabajar que tienen } \\
\text { los científicos. En este caso, tienen que } \\
\text { aprender que los científicos observan el } \\
\text { sistema óseo del ser humano y para } \\
\text { hacerlo más sencillo y comprensible para } \\
\text { los demás, clasifican las características de } \\
\text { sus elementos en función de las } \\
\text { semejanzas, pudiendo así agruparlos.» }\end{array}$ & $\begin{array}{l}\text { «Quiero que el alumnado sea capaz de } \\
\text { analizar los huesos y de pensar cuales son } \\
\text { las características que comparten entre } \\
\text { ellos para, posteriormente, poder } \\
\text { clasificarlos de una forma u otra.» }\end{array}$ \\
\hline $\begin{array}{l}\text { ¿Por qué es } \\
\text { importante para el } \\
\text { alumnado } \\
\text { aprender estos } \\
\text { contenidos? }\end{array}$ & $\begin{array}{l}\text { «Es imprescindible que el alumnado sepa } \\
\text { que los científicos trabajan de manera } \\
\text { ordenada y coherente para, así, } \\
\text { facilitarnos la comprensión de los } \\
\text { contenidos.» }\end{array}$ & $\begin{array}{l}\text { «Es primordial que el alumnado sea capaz } \\
\text { de establecer distintos criterios de } \\
\text { clasificación en función de los elementos } \\
\text { que está estudiando o analizando puesto } \\
\text { que, saber categorizar no solo le va a ser } \\
\text { útil en el ámbito científico, sino que en } \\
\text { cualquier asignatura académica o ámbito } \\
\text { de su vida cotidiana la categorización } \\
\text { puede estar presente.» }\end{array}$ \\
\hline $\begin{array}{lr}\text { ¿Cuáles son } & \text { los } \\
\text { métodos } & \text { para } \\
\text { evaluar } & \text { la } \\
\text { comprensión } & y \\
\text { desarrollo de estos } \\
\text { contenidos? }\end{array}$ & $\begin{array}{l}\text { «Esta idea sobre la naturaleza de la ciencia } \\
\text { la evaluaremos a través de preguntas } \\
\text { presentadas en la situación de enseñanza- } \\
\text { aprendizaje: ¿Por qué creéis que los } \\
\text { científicos clasifican los huesos del } \\
\text { cuerpo humano según su tamaño? } \\
\text { ¿Resulta eficaz? Justifica la respuesta.» }\end{array}$ & $\begin{array}{l}\text { «Para evaluar esta destreza de } \\
\text { pensamiento se formularían las siguientes } \\
\text { preguntas, también presentadas en la } \\
\text { situación de enseñanza-aprendizaje: ¿Sois } \\
\text { capaces de explicar en qué os habéis } \\
\text { basado para montar el esqueleto? ¿Qué } \\
\text { criterio habéis seguido?»» }\end{array}$ \\
\hline
\end{tabular}

Tabla 2. Reflexiones de un estudiante sobre la intención e importancia didáctica y métodos de evaluación de un rasgo de la $\mathrm{NdC}$ en el primer ciclo de Educación Primaria.

\section{Rasgo de la NdC}

Para mejorar la comprensión de la naturaleza, los/as cientificos/as elaboran esquemas de clasificación.

\section{Respuesta}

¿Qué pretendes que aprendan los estudiantes sobre estos contenidos?

¿Por qué es importante para el alumnado aprender estos contenidos?

¿Cuáles son los métodos para evaluar la comprensión y desarrollo de estos contenidos?
«Es fundamental una buena estructuración de los contenidos para facilitar el aprendizaje.»

«Para retener la información de una manera más eficaz los estudiantes tienen que crear esquemas de clasificación que sean fáciles de comprender para ellos mismos.»

«La realización por parte de los estudiantes de un esquema de la información dada.» 